\title{
Evaluation of Associated Motion Parameters of Descending Moon Impact Probe (Mip) of Chandrayan-1 From Time Sequential Images
}

\author{
Vinay K Srivastava ${ }^{1, *}$, Tapas Kumar $\operatorname{Nandy}^{1}$, Anurag Verma ${ }^{2}$ \\ ${ }^{1}$ Dept of Applied Geophysics, Indian School of Mines, Dhanbad , India \\ ${ }^{2}$ Space Applications Centre (ISRO), Ahmedabad, India \\ *Corresponding Author: ismkvinay@gmail.com
}

Copyright (C) 2013 Horizon Research Publishing All rights reserved.

\begin{abstract}
The MIP, a micro satellite probe onboard Chandrayaan -1 was designed to impact on moon surface at pre decided location for scientific data collection from close range which was released from Chandrayaan-1 mother ship on $14^{\text {th }}$ November 2008. This MIP impacted near the moon surface near south-pole after 24 minutes from its release and while descending MIP suffered a composite motions consisting of three fundamental motions viz. spinning, coning and forward translational (velocity). Such descending MIP showed a definite pattern of coverage on moon surface. Therefore in order to monitor and to select future landing site of impact on moon surface the associated component of motion viz.; spinning rate, coning rate, velocity of descend of MIP needs to be calculated and known. In view of this, an attempt has been made here to derive associated parameters of composite motions and attitude of MIP for predicting future impact site of descending probe on moon surface from the orbiting space craft using the time sequential images acquired by the descending MIP and the algorithm developed under MATLAB after Srivastava et. al. 2011.
\end{abstract}

Keywords Chandrayaan1, Moon Impact Probe, MATLAB, South Pole

\section{Introduction}

Moon Impact Probe (MIP), a micro satellite probe was the most important payload among the 11 payloads onboard Chandrayaan-1 designed by the Indian Space Research Organisation (ISRO) to impact the Shackleton Crator area near to south pole region of moon (Bussey et al 1999). The MIP took about 24 min to impact on the moon surface after it's separation from the mother ship Chandrayaan-1 (Fig.1) consisting of three other instruments viz.; Radar Altimeter, Mass Spectrometer and Video Imaging System ( Kumar 2009,Goswami 2009) and while descending MIP has shown a definite pattern of coverage on moon surface.

The mission sequence of the MIP is given as:

$\mathrm{T}_{0}-20 \mathrm{~min} \quad$ MIP Power on

$\mathrm{T}_{0}-5 \mathrm{~min} \quad$ MIP Enable Command

$\mathrm{T}_{0} \quad$ MIP separation

$\mathrm{T}_{0}+10 \mathrm{sec} \quad$ Spin up of MIP

$\mathrm{T}_{0}+1489.02 \mathrm{sec}$ MIP impact

In present study the concept of fundamental motions of descending MIP has been applied for extracting individual motion parameters such as spinning rate, coning rate and velocity from the acquired sequential images of descending MIP over moon surface using the developed algorithm under MATLAB after Srivastava et al, 2011.

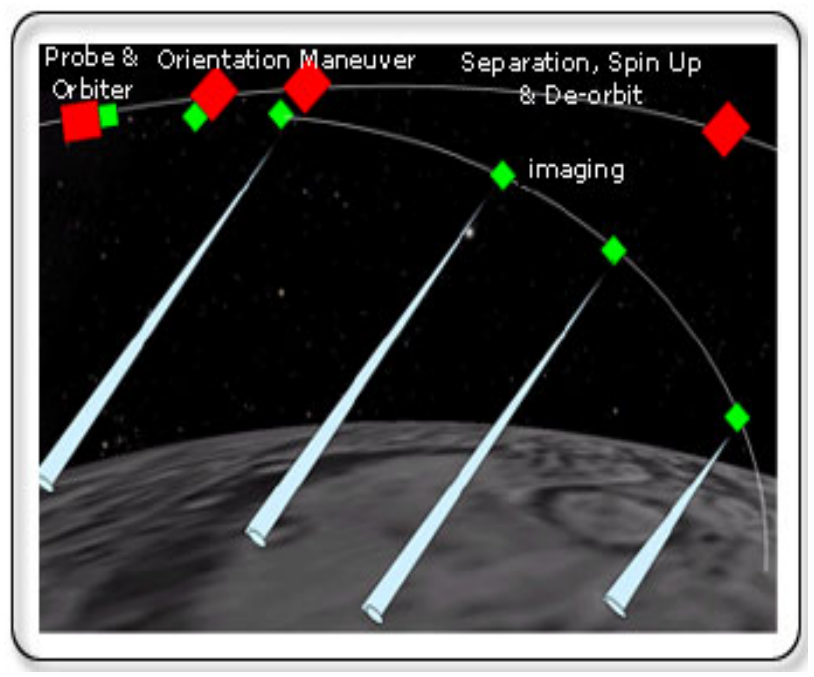

Figure 1. MIP Mission and path (Kumar ,2009)

\section{Data and Methodology Used}

In present study we have used near about 3000 - time sequential MIP photo images (720x576 pixels), acquired after the 25 minutes journey at 20:31 Hrs IST (Indian 
Standard Time) covering the area around Shackleton Crater located in south polar region of moon and were received on 14th of November 2008 by Chandrayaan-1. The time interval between two subsequent odd-even frames has been $20 \mathrm{~ms}$ whereas it is $1.7 \mathrm{sec}$ in between two subsequent even-odd frames ( Kumar 2009). These MIP images have shown a definite pattern of coverage on moon surface under the influence of composite motions of descending probe and from this pattern an attempt has been made to derive composite motions and the attitude parameters of MIP viz.; spinning rate, coning rate, velocity and inclination using algorithm developed after Srivastava et al., 2011.

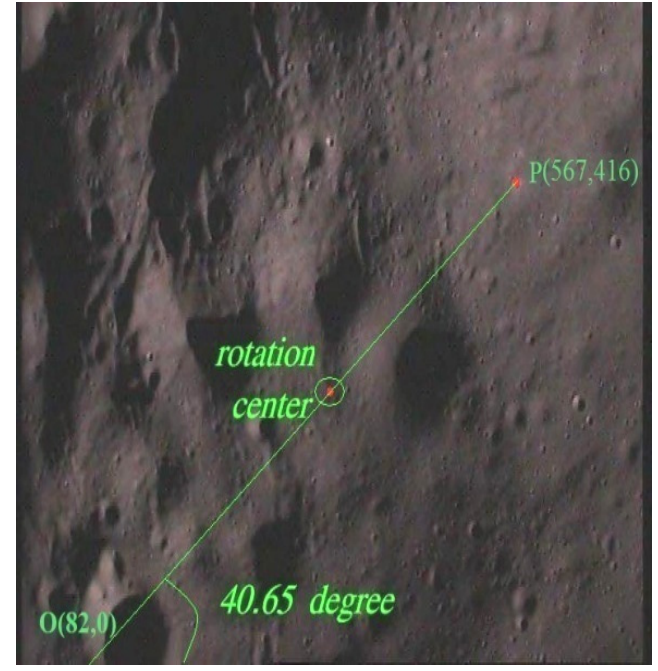

Frame\#VIS2899

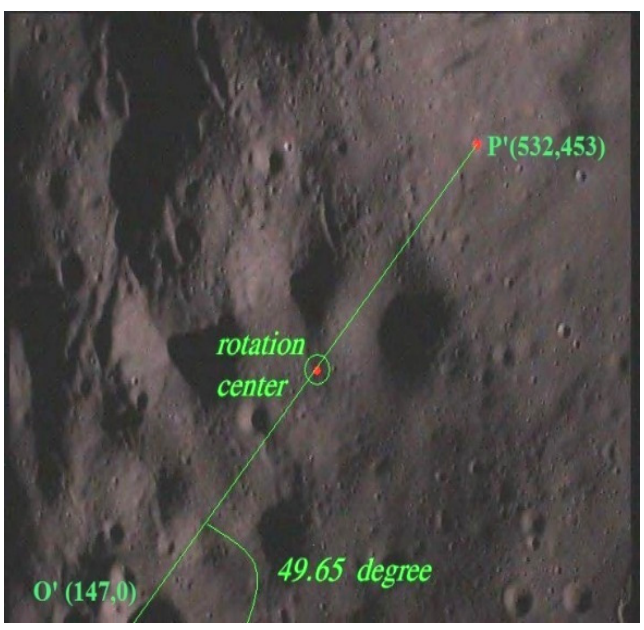

Frame \# VIS2890

Figure 2. Showing two subsequent odd and even frames.

\section{Analysis and Discussions}

\section{A. Spinning Rate}

For calculating this rate two subsequent odd-even frames \#VIS 2899 and \#VIS 2900 ( Fig. 2) with time interval $20 \mathrm{~ms}$ have been taken where the point of rotation center has not changed and so by taking a common feature in both the frames one calculates the angle swept by the feature with respect to the center of rotation in a given interval of time. However the later frames of MIP ( Fig. 3) have been taken when the view of footprints of MIP are almost vertical and so the distortion is minimum due to inclination.

In above two frames the $\mathrm{P}$ and $\mathrm{P}$ ' features are same. In frame \#VIS 2899, the pixel co-ordinate of $\mathrm{P}$ is $(567,416)$ and the extended line from the rotation center cut the $\mathrm{x}$-axis at point $\mathrm{O}(82,0)$. So the angle $(\Phi)$ subtended by point $\mathrm{P}$ with the $\mathrm{x}$ - axis is given by equation 1 ,

$$
\Phi=\tan ^{-1}(416 / 567-82)=40.65^{\circ}
$$

Similarly ) in frame VIS \# 2900 for point P' $(532,453$, the angle $\left(\Phi^{\prime}\right)$ is given by the equation $2 . \Phi^{\prime}=\tan ^{-1}$

$$
(453 / 532-147)=49.65^{0}
$$

This means the features subtends an angle of $9^{0}$ $\left(=49.65^{\circ}-40.65^{\circ}\right)$ in $0.02 \mathrm{sec}$ of time interval and in this fashion it completes one rotation in $0.8 \mathrm{sec}(=0.02 * 360 / 9)$ which gives a spinning rate equivalent to 75 rotation per minute (RPM).

\section{B. Coning Rate}

The spinning motion of MIP causes in a definite pattern of coverage on moon surface where as the coning motion causes the features to reappear after some time in a set of images taken by the MIP. This could be seen clearly in initial frames where size of footprint is very large which reduces subsequently near the impact site ( Fig. 3) and thus possibility of revisiting of an object during one coning cycle is minimal. This is due to fact that the continued forward motion of camera displaces it away from earlier site before camera points back at the end of one coning cycle.

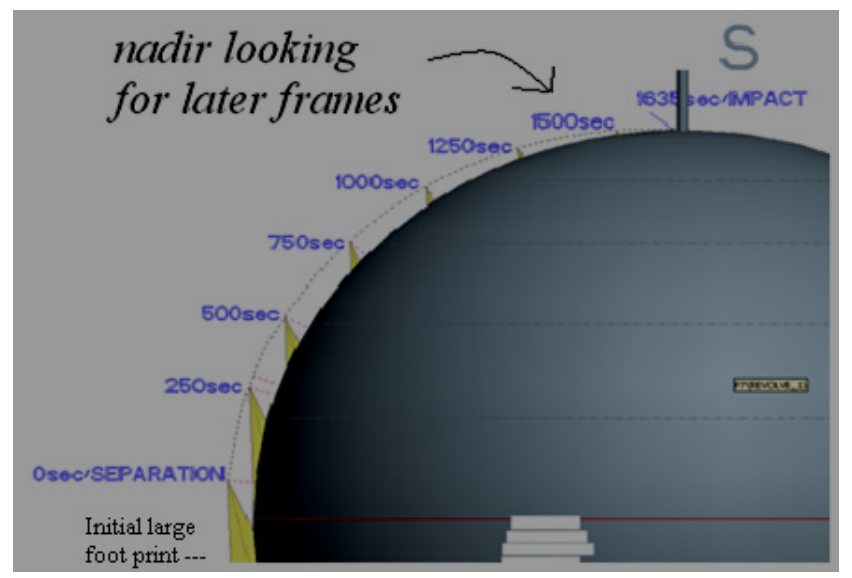

Figure 3. Variation in size of foot prints of MIP with inclination after separation.

Reappearing objects have been observed and identified on a set of images and the time taken by that object to move from one extreme to the other and then return to its original position has been taken as one coning cycle period. Thus the frequency of coning cycle provides the coning rate of MIP motion. In the images shown below (Fig. 4) it is observed 
that the features reappear / reorient themselves exactly after 20 frames (ie 10 pairs of even and odd frames).

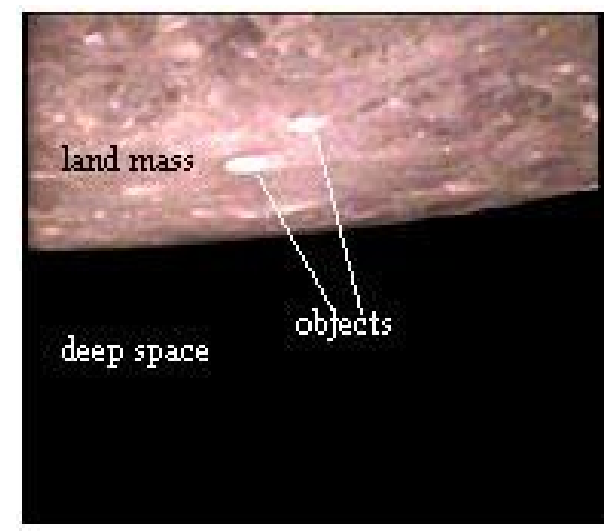

Frame \# VIS 1405

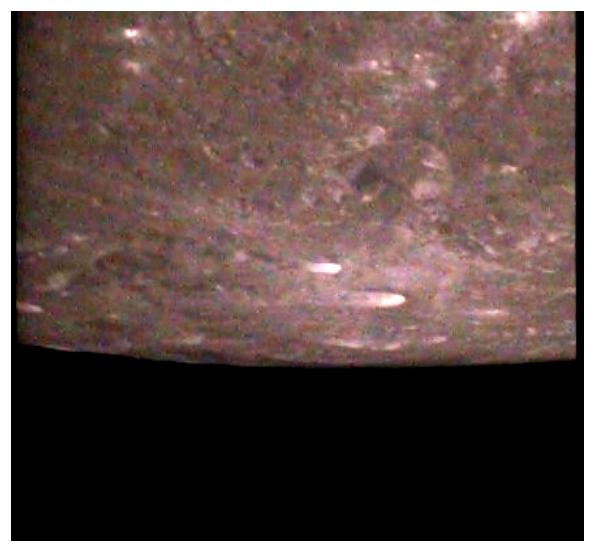

Frame \# VIS 141

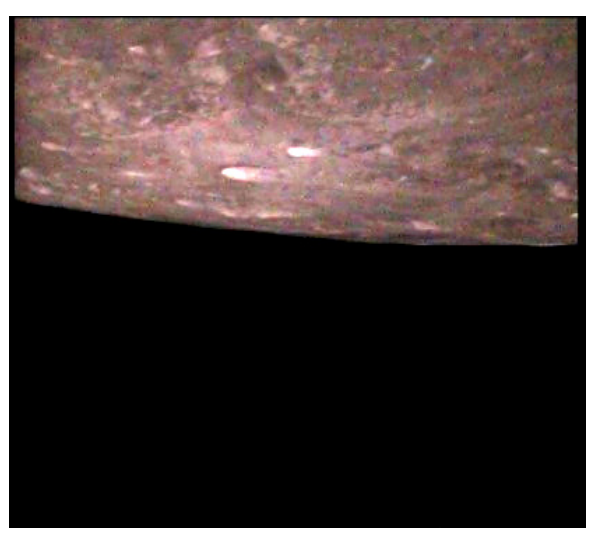

Frame \# VIS 1425

Figure 4. Shows reappearance of objects exactly after 20 frames (\#VIS 1405 -\#1425)

In initial frame $\# 1405$, where the edge of landmass is almost horizontal which rotates itself by almost $180^{\circ}$ after 10 frames ie in frame \# 1415. Finally the edge reorients as per the frame \#1405 exactly after another 10 frames ie in frame \# 1425 , however, with some residual angel. In above three it also is observed that the ratio of deep space to land mass is changing from frame to frame and similar pattern is repeated after 20 frames.

Now the time offset between 20 frames is $1.7 * 10+$ $0.02 * 10=17.2 \mathrm{sec}$ which gives rotation speed equivalent to 0.05814 rotation per second (RPS) and spinning rate equivalent to 3.485 . This directly converts to a coning rate equal to 3.5 RPM (approximately) or its multiple which is the minimum possible value of coning rate. However it is not possible to measure accurately the rotation angle due to coning motion because it is possible to observe the effect of residual angle only at the end and hence it is not possible to know the number of rotation it has completed before it has reached to present position with residual angle and numbers of registered frames. Thus the minimum value of conning rate is 3.5 RPM approximately.

\section{Velocity-Forward Translational Motion}

For this we have taken two frames (\# VIS 2871 and \#VIS 2874) where the object $(\mathrm{P})$ has appeared and the view of the foot prints of MIP are nearly vertical with angle is less then $4^{0}$ as shown in Fig.5.

Table 1. Details of Frames

\begin{tabular}{|c|c|c|c|c|}
\hline Frame \# & $\begin{array}{c}\text { View } \\
\text { angle } \\
(\text { degree })\end{array}$ & $\begin{array}{c}\text { Altitude } \\
(\mathrm{km})\end{array}$ & $\begin{array}{c}\text { Foot print } \\
\left(\mathrm{Km}^{2}\right)\end{array}$ & Pixel no. \\
\hline 2871 & 2.3297 & 21.18 & $10.93 \times 8.44$ & $720 \times 576$ \\
\hline 2874 & 2.2776 & 20.97 & $10.82 \times 8.36$ & $720 \times 576$ \\
\hline
\end{tabular}

Here the total time difference $(\mathbf{t})$ between these two frames is 1.74 secs as explained below. \#2871--20 msec---- \# 2872---. ${ }^{1.7 \mathrm{sec}}$--- \# 2873-- $20 \mathrm{msec}$

So the elapsed time in between the above two frames is $1.74 \mathrm{sec}$ and by taking the calculated coning rate as 3.5 RPM $(=0.05833$ rotation per second $)$ and the spinning rate as 72 RPM (= ie 1.2 rotation per second) the total rotation comes out to be $36.42^{\circ}$ and the total rotation angle due to spin equals to $751.68^{\circ}\left(=1.2^{*} 1.74\right)$. Thus the total rotation due to coning and spinning comes out to be $88.1^{0}\left(=36.42^{0}+751.68^{\circ}\right)$ which could be attributed to two full rotation $\left(=720^{\circ}\right)$ plus a residual angle of $68.1^{0}$.

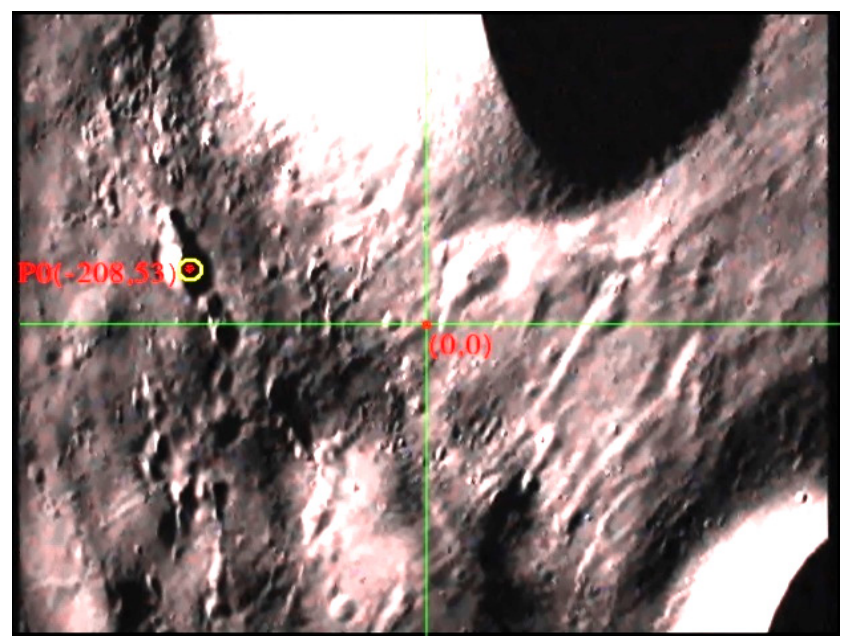

Frame \# VIS 2871 


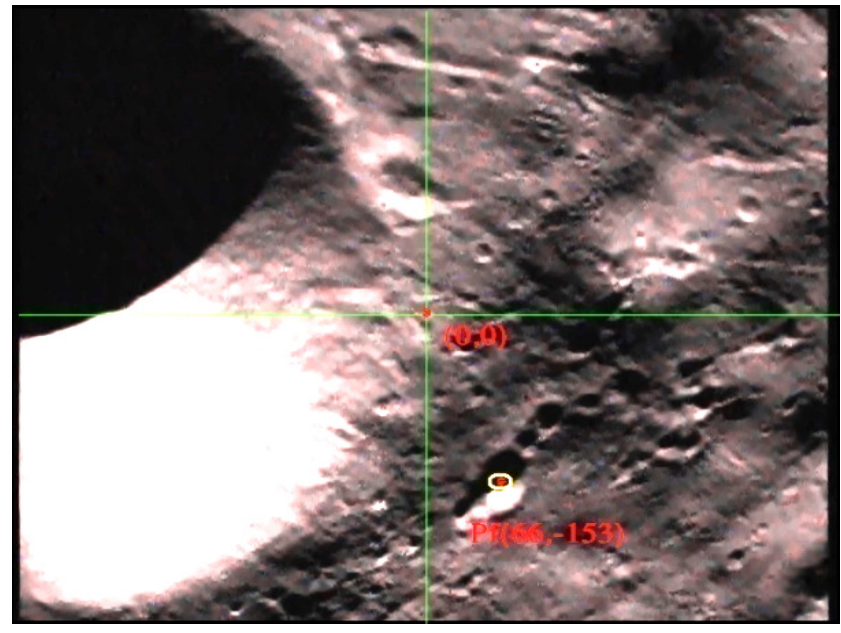

Frame \# VIS 2874

Figure 5. $\quad \mathbf{P}_{0}$ and $\mathbf{P}_{\mathrm{f}}$ shows the position of objects in frame \#VIS 2871 and \# VIS 2874 respectively with almost vertical view due to coning and spinning motions of MIP.

In frame \# VIS 2871, the co-ordinate of the feature is $\mathbf{P}_{\mathbf{0}}\left(\mathbf{x}_{\mathbf{0}}, \mathbf{y}_{\mathbf{0}}\right)=(-208,53)$ and in the frame \# VIS 2874 the co-ordinate of final position is $\mathbf{P}_{\mathbf{f}}\left(\mathbf{x}_{\mathbf{f}}, \mathbf{y}_{\mathbf{f}}\right)=(66,-153)$ and such displacement in position of features has happened due to composite motions of MIP viz. the spinning, coning, and translational.

Therefore by applying the rotational matrix $\mathrm{R}$ with residual $(\theta)=68.1^{0}$ on the initial co-ordinate $\mathbf{P}_{\mathbf{0}}$, one get the amount of displacement due to the spinning and coning only. The difference between the final position, $\mathbf{P}_{\mathbf{f}}$ and the position after rotation shall give the effect of the velocity component only for which the pixel coordinate has comes out to be , $\mathbf{P}_{\mathbf{s}}(-127.05,-172.95)$.

This point $\mathbf{P}_{\mathbf{s}}$ represents the position of initial point, $\mathbf{P}_{\mathbf{0}}$ $\left(\mathbf{x}_{\mathbf{0}}, \mathbf{y}_{\mathbf{0}}\right)$ under the influence of two motions of MIP viz.; spinning and coning without the effect of velocity. Therefore the difference in position in between the point $\mathbf{P}_{\mathbf{s}}$ and the final point $\mathbf{P}_{\mathbf{f}}$ shall give translational component of initial point features due to the velocity. This distance (d) between these two points $\mathbf{P}_{\mathbf{s}}$ and $\mathbf{P}_{\mathbf{f}}$ is approximated to be around 194 pixel as derived as shown below using equation (3).

$$
\begin{aligned}
\mathrm{d}=\sqrt{ }\left(\left(\mathrm{x}_{\mathrm{f}-\mathrm{x}} \mathrm{x}^{\prime}\right)^{2}+\left(\mathrm{y}_{\left.\left.\mathrm{f}-\mathrm{y}^{\prime}\right)^{2}\right)}\right.\right. & \\
& =194.1 \text { pixels }
\end{aligned}
$$

and the rotational angle between these two points is given by equation (3).

$$
\alpha=\tan ^{-1}\left(\mathrm{y}_{\mathrm{f}-\mathrm{y}} \mathrm{y}^{\prime}\right) /\left(\mathrm{x}_{\mathrm{f}-\mathrm{x}} \mathrm{x}^{\prime}\right)=5.92^{0}=6^{0} \text { approx }
$$

The distance, $\mathrm{d}$, is the distance between the final point $\mathbf{P}_{\mathbf{f}}$ and the point $\mathbf{P}_{\mathbf{s}}$ after rotation due to the spinning and coning. However we know that the MIP has travelled a distance in a time interval, a translational (forward) motion giving the velocity of motion. Hence the distance travelled by the object in the frames is only due to velocity and the calculation of which is described below:

Taking the reported velocity value of MIP as 1.6363 $\mathrm{km} / \mathrm{sec}$ (Kumar 2009), the distance travelled by the MIP comes out to be $2.8471 \mathrm{~km}$. in time interval of $1.74 \mathrm{sec}$ and then considering the average foot print size $(720 \times 576)$ pixels the corresponding size of one pixel equals to $0.015 \mathrm{~km} \mathrm{(=}$ $10.85 \mathrm{~km} / 720)$ on moon surface. Thus in fact the travelled distance (d) in terms of pixel dimension (p) is 189.8 pixels.

Thus from above calculation we observed that the camera in a given time has moved from point $\mathbf{P}_{\mathbf{s} \text { to }} \mathbf{P}^{\prime}$ and is 189.8 pixel away. However the calculated distance in terms of pixel between the shifted position, $\mathbf{P}_{\mathbf{s}}$ and the final position, $\mathbf{P}_{\mathbf{f}}$ has come out to be 194.1 pixels with orientation of motion vector as $6^{0}$. But after de rotating the images (Fig. 6) the angel of orientation of motion vector comes out be $57^{\circ}$.

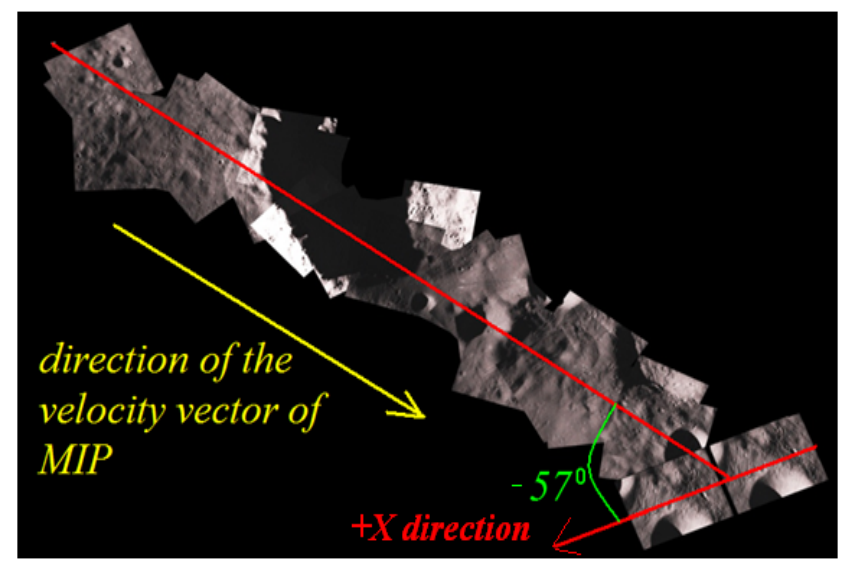

Figure 6. The space sequential images and direction of motion vector after de-rotation.

Now we are observing that the direction of velocity vector is not matching with the value derived from present study. Probably we have considered small value of coning rate which may be multiple of cycle and so the sampling rate is very coarse which is not adequate to resolve the accurate number of rotation (n).

\section{Conclusions}

Using the time sequential images acquired by the descending probe and the resulting pattern of surface cover on moon it has been possible to estimate parameters of motion components associated with descending MIP viz.; the spinning motion, coning motion and velocity (forward translation motion ) which are described below:

1. From the sequential images, the spinning rate of the MIP has been obtained as 75 RPM where as the system Accelerator has provided a value of 72 RPM .

2. The derived value of conning rate of MIP is 3.5 and its multiples. It has become difficult to determine distinct number of multiples as because the numbers of registered frames are not adequate to calculate this.

3. The distance travelled by the MIP is $2.9115 \mathrm{~km}$ $(194.1 * 0.015)$ which gives a velocity equivalent to 1.6732 $\mathrm{km} / \mathrm{sec}$ as against the reported $1.6363 \mathrm{~km} / \mathrm{sec}$. velocity due to reason as explained above.. 
Such discrepancies in calculated motion parameters are probably occurring due to association of an external force which modifies the motion of MIP and nature of which is to be ascertained.

\section{Acknowledgments}

Authors express their sincere thanks to the Director, Space Application Centre (ISRO) Ahmedabad, India and also to Director, Indian School of Mines, Dhanbad, India for providing required facilities to carry out above work. Junior author (TKN) in particular is thankful to Scientists of Space Application Centre, Ahmedabad for permitting him to work as Intern on this problem.

\section{REFERENCES}

[1] Bussey DBJ, BD Spudis and MS Robinson (1999), "Illumination condition at the Lunar South pole", Geophysics Research Letter ,26, pp 1187-1190.

[2] Goswami J.N., M. Annadurai, (2009), "Chandrayaan-1: India's first planetary science mission to the moon," Current Science, vol. 96, no.4. pp.486-491

[3] Kumar Ashok and MIP Project Team, ( 2009), "The Moon Impact Probe on Chandrayan -1", Current Science, Vol 96, No 4 Feb 2009 pp 540 - 543.

[4] Srivastava V K, Nandy T K and Verma A, (2011), "Study of motion components associated with descending Moon Impact Probe (MIP) to moon surface from the chandrayaan-1 space craft from simulated surface motion traces using the developed algorithms under MATLAB and from sequential photo images acquired during the descend", Int. Journal of Experimental Algorithm, Vol 2, Issue 3,pp 74-81. 